\title{
Preparation and in Vitro Dissolution of Curcumin Tablets
}

\author{
Yuanping Wang ${ }^{a}$, Chuqin $\mathrm{Yu}^{*}$, , Zhixiang Gan $^{\mathrm{c}}$, Zhongbo Xie ${ }^{\mathrm{d}}$ \\ Guangdong Pharmaceutical University, Guangzhou, 510006, China \\ ayuanpingwang@163.com, ${ }^{\mathrm{b} p n 333 @ 163 . c o m(c o r r e s p o n d i n g ~ a u t h o r), ~ c g a n z h i x i a n 025 @ 163 . c o m, ~}$ \\ dzhonb0507@163.com
}

Keywords: Curcumin; dissolution; tablets; preparation process; response surface methodology

\begin{abstract}
Objectives: To prepare curcumin tablets, and evaluate their in vitro dissolution. Methods: Curcumin was dissolved in acetone, wet-granulation and tableting. The amount of Poloxamer 188 and Gremophor RH40 were chosen as factors, and the in vitro dissolution at 120 min was used as evaluation index. The best prescription was screened by response surface method of two factors and three levels. UV-visible spectrophotometry was used as detection methods. Results: In this study, the standard curve was in good linearity with the range of $2.5 \sim 5.0 \mu \mathrm{g} \cdot \mathrm{mL}^{-1}, \mathrm{r}=0.9996$. The average recovery was $98.08 \%$, RSD $=0.61 \%$. The obtained prescription: Curcumin: Poloxamer 188: Gremophor RH40 = 1: 2.52: 0.40 , the in vitro dissolution at $45 \mathrm{~min}$ and $120 \mathrm{~min}$ were $64.85 \%$ and $95.12 \%$ respectively. Dissolution of the best prescription of curcumin obtained twice higher than the raw curcumin and physical mixtures, and higher than commercial products. Conclusions:The preparation method of curcumin tablets is simple and reliable, which significantly improves the dissolution of curcumin.
\end{abstract}

\section{Introduction}

Curcumin which was medicinal and edible products was mainly from the root of Curcuma aromatica Salisb, turmeric roots, Curcuma Rhizome, Acorus calamus L roots, etc. In medicine it has antioxidant, anti-inflammatory, anti-proliferative, anti-cancer, anti-bacterial infection, liver damage and other effects protection [1]. In recent years, it has been found to have immunomodulatory effect and a significant effect on the prevention of Alzheimer's disease[2]. Due to its low toxicity (LD50 in mice was greater than $2 \mathrm{~g} . \mathrm{kg}^{-1}$ [3]), the US FDA had evaluated its extensive safety. However, curcumin has some shortcomings such as poorly dissolution in water, sensitive to light, heat and iron ion, easily hydrolyzed in neutral and alkaline environment, content declining sharply under outdoor lighting [4], decreasing after autoclaving, low oral bioavailability [5] etc. Various preparation methods were reported in literature which mainly used polyvinylpyrrolidone (PVP K30), polyethylene glycol (PEG) as carriers to prepare curcumin solid dispersion in order to improve its dissolution [6-8], but the preparation process was complicated and high cost, which was not suitable for large-scale production. In this study, the response surface methodology (RSM) was used to obtain the best preparation of curcumin tablets, thus to increase its dissolution and bioavailability as well as to simplify the production process and facilitate the large-scale production and lower production costs.

\section{Reagent and Instrument}

Curcumin substance(Cur, Hebei days Asahi natural pigment Ltd.); Curcumin reference (Guangzhou Qiyun Sheng Technology Co., Ltd., content: HPLC $\geq 98 \%$ ); Poloxamer 188 (P188, BASF); Gremophor RH40 (Gremophor RH40, BASF); Crosslinked povidone (PVPP, the United States Specialty Products company); Silica powder $\left(\mathrm{SiO}_{2}\right.$, Guangzhou Jie Fu trading Co., Ltd.); Low-substituted hydroxypropyl cellulose (L-HPC, Guangzhou Jie Fu trading Co., Ltd.); Lactose (Beijing Feng Jingqiu commercial limited liability company); Microcrystalline cellulose (MCC, Anhui mountains and rivers of medicinal materials Co., Ltd.); Magnesium stearate (MS, Guangzhou Jie Fu trading Co., Ltd.); Curcumin capsules (US Priscilla Pleasant company); Sodium chloride (NaCl, Tianjin Zhiyuan chemical Reagent Co.); Sodium dodecyl sulfate (SDS, Tianjin Zhiyuan Chemicals Limited); Acetone (Tianjin Damao Chemical Reagent Factory). 
ZRS-8G Intelligent Dissolution Tester (Tianjin Tianda Tianfa Technology Co., Ltd.); ZKT-18F vacuum degassing device (Tianjin Tianda Tianfa Technology Co., Ltd.); GZX-9246 MBE Digital Blast Oven (Shanghai Bo Xun Industrial Co., Ltd. medical Equipment Factory); UV-1800 UVvisible spectrophotometer (Shimadzu Corporation, Kyoto, Japan); BS 224S electronic balance (Beijing Sartorius instrument systems, Inc.); YP3001N electronic balance (Shanghai precision Scientific instruments Ltd.); ZP-5B rotary tablet machine (Shanghai Tianfeng pharmaceutical Machinery Co.); RCT magnetic stirrer (Germany IKA company); KQ-100 ultrasonic cleaner (Kunshan Ultrasonic instrument Co., Ltd.); TDL-60B desktop centrifuge (Shanghai Anting scientific Instrument Factory); MA35 electronic moisture Analyzer (Guangzhou Great Technology instrument Co., Ltd.).

\section{Methods and Results}

\section{Study on the Dissolution Method Preparation of the Solution}

Preparation of the Dissolution Medium

Take $11.7 \mathrm{ml}$ hydrochloric acid into $50 \mathrm{ml}$ volumetric flask, dilute with water to the mark, to obtain hydrochloric acid. Take about $2 \mathrm{~g} \mathrm{NaCl}$ and SDS respectively, with precise metage, adding the above hydrochloric acid $16.4 \mathrm{ml}$, and diluted into $1000 \mathrm{ml}$ with water, degassing, then obtain the dissolution medium.

Preparation of the Standard Solution

About $10 \mathrm{mg}$ of Cur standard, with precise metage, was placed in a $500 \mathrm{ml}$ brown volumetric flask, adding $20 \mathrm{ml}$ of $95 \%$ ethanol, ultrasonic $5 \mathrm{~min}$, then cooled, with dissolution medium to the scale, finally obtained standard solution.

Tested Sample Solution Preparation

Take 20 curcumin tablets, grinded into fine. About $200 \mathrm{mg}$ of fine powder, was placed in a $500 \mathrm{ml}$ brown volumetric flask, adding proper amount of $95 \%$ ethanol, ultrasonic $5 \mathrm{~min}$, then cooled,with dissolution medium to the scale.

\section{UV Absorbance Wavelength Selection}

Measured standard solution $5 \mathrm{ml}$ and placed it into $20 \mathrm{ml}$ brown volumetric flask, with dissolution medium to the scale, filtered, and took successive filtrate, Geometric (V/V) ethanol dissolution medium was used as blank control in the wavelength range of 300-500nm scanning. Cur in $429 \mathrm{~nm}$ had maximum absorbance, so wavelength of $429 \mathrm{~nm}$ was selected for Cur maximum absorbance.

\section{Membrane Adsorption Test}

According to the method of dissolution determination,one piece of curcumin tablets was taken into the dissolution cup, and the samples were taken for 9 copies, each $10 \mathrm{ml}$. Three of them were placed in the $10 \mathrm{ml}$ centrifuge tube, with the rotating speed of $5000 \mathrm{r} \cdot \mathrm{min}^{-1}$, centrifuged for $15 \mathrm{~min}$. The dissolution medium was used as the blank control,and theabsorbance of the supernatant was measured at the wavelength of $429 \mathrm{~nm}$. The remaining six copies all passed over $0.8 \mu \mathrm{m}$ micropore filter membrane, three of which were abandoned $2 \mathrm{ml}$ of the initial filtrate, while the other three were abandoned $3 \mathrm{ml}$ of the initial filtrate. Take the successive filtrate and the dissolution medium as blank control respectively and determine the absorbance at the wavelength of $429 \mathrm{~nm}$. Results showed that the average value of $2 \mathrm{ml}$ membrane adsorption capacity was $1.96 \%$, and $3 \mathrm{ml}$ was $0.03 \%$, which were less than $2 \%$.

\section{Standard Curve}

The standard solution was diluted with the dissolution medium to a series concentrations of solutions of $2.5,3.0,3.5,4.0,4.5$ and $5.0 \mu \mathrm{g} \cdot \mathrm{ml}^{-1}$. Filter and take the successive filtrate with geometric ethanol dissolution medium as blank control, determinate the absorbance at the wavelength of $429 \mathrm{~nm}$. The concentration $\mathrm{C}$ used as $\mathrm{y}$ axis on the ceiling light value for $\mathrm{X}$ axis for linear regression, the regression equation was as follows $\mathrm{C}=0.0072 \mathrm{~A}+0.00005$, the related coefficient $\mathrm{r}=0.9996$. The results showed that the linear relationship was good in the range of 2.5 to $5 \mu \mathrm{g} \cdot \mathrm{ml}^{-1}$. 


\section{Precision Test}

According to the determination method of dissolution, take one piece of curcumin tablet into a dissolution cup, sample $60 \mathrm{ml}$ at $45 \mathrm{~min}$, filter, take the successive filtrate, the dissolution medium as blank control, determine the absorbance at the wavelength of $429 \mathrm{~nm} 6$ times within one day, calculate the concentrations, $\mathrm{RSD}=0.065 \%$. The results showed that the precision of the instrument was good.

Stability Test

According to the determination method of dissolution, take one piece of curcumin tablet into a dissolution cup, sample $60 \mathrm{ml}$ at $45 \mathrm{~min}$, filter, take the successive filtrate, and the filtrate was divided into 7 parts. Take the dissolution medium used as the blank control and determinate the absorbance at the wavelength of $429 \mathrm{~nm}$ at $0,1,2,3,4,5$ and $6 \mathrm{~h}$, respectively,then calculate the concentrations, $\mathrm{RSD}=0.320 \%$. The results showed that the stability of solution was good within 6 hours.

\section{Excipients Interference Test}

According to the preparation process and the amount of the prescription, make blank tablets without drugs. Take $20 \mathrm{mg}$ of blank tablet and grind them into fine, take about $10 \mathrm{mg}$ of fine powder, with precise metage, place it in a $25 \mathrm{ml}$ brown volumetric flask, with $95 \%$ ethanol and diluted to the scale, ultrasonic $5 \mathrm{~min}$, chilling, take $2 \mathrm{ml}$ into a $10 \mathrm{ml}$ volumetric flask, diluted to the scale with dissolution medium, shake, filter, take the successive filtrate. Take dissolution medium containing geometric ethanol $(\mathrm{V} / \mathrm{V})$ as blank control and determine the absorbance at the wavelength of $429 \mathrm{~nm}$, and scan in the wavelength range of $300 \sim 500 \mathrm{~nm}$. The results showed no absorption peak at $429 \mathrm{~nm}$, which indicated no interference from excipients.

\section{Recovery Test}

Take 3 copies of standard solutions of $2.5,4,5 \mathrm{ml}$ respectively into $10 \mathrm{ml}$ brown volumetric flask, add fine powder of the blank tablets with amount of prescription, dilute with dissolution medium to the sample solutions containing 50\%,80\%,100\% Cur, Filter the above solution, take successive filtrate, and use dissolution medium of corresponding concentration of ethanol as blank control to determinate the absorbance at the wavelength of $429 \mathrm{~nm}$. Result showed that the average recovery rate was $98.08 \%$, RSD $=0.610 \%$, which was in line with the requirements.

\section{In Vitro Dissolution Test}

Take this product, according to the dissolution test method (Appendix X C second), with hydrochloric acid solution (containing $0.2 \% \mathrm{SDS}$ and $0.2 \% \mathrm{NaCl}, \mathrm{w} / \mathrm{v}$ ) as the dissolution medium at the speeding rate of 50, According to the operation, sample $10 \mathrm{ml}$ at 45, 60, 90 and $120 \mathrm{~min}$, then supplement fresh medium of the same temperature and volume, filter, take successive filtrate, take precisely moderate amount of successive filtrate, diluted quantitatively with dissolution medium to obtain the Cur concentration of $3 \mu \mathrm{g} \cdot \mathrm{ml}^{-1}$. According to UV-VIS spectrophotometry (Appendix IV A), the absorbance is determinated at the wavelength of $429 \mathrm{~nm}$. And then take another Cur standard solution, dilute it to $3 \mu \mathrm{g} \cdot \mathrm{ml}^{-1}$. The absorbance was determined by the same method, and the dissolution amount of each table was calculated.

\section{Preparation of Curcumin Tablets}

Prescription amount of L-HPC, lactose and MCC were weighed and screened through No.5 mesh sieve respectively, and then mixed equably and standby. Prescribed amount of Poloxamer 188 was added into an appropriate amount of acetone, stirred until completely dissolved. Add prescriptions amount of Cur, and mix well. Then add Gremophor RH40, stir until Cur was completely dissolved. Prescription amount of PVPP and $\mathrm{SiO}_{2}$ were added to the above solution with stirring. The backup mixture flour was added to the system, and soft material was prepared and screened a No.2 mesh sieve to granulate. The granules were dried at $40{ }^{\circ} \mathrm{C}$ to a control the moisture content at $3 \sim 6 \%$, over a NO. 2 mesh sieve. a prescription amount of lubricant $(0.4 \%$ MS calculated by particles $(\mathrm{W} / \mathrm{W}), 0.6 \%$ $\mathrm{SiO}_{2}$ calculated by particles $(\mathrm{W} / \mathrm{W})$ ) was added and mixed well. Measure content and prepare tablets. The weight of every tablet is about $200 \mathrm{mg}$.

\section{Preparation Process Optimization of Curcumin Tablets}

Previous studies found that a certain amount of Poloxamer 188 and Gremophor RH40 caused tableting process sticking phenomenon, and affected the disintegration and dissolution of the tablets. 
Therefore, Poloxamer 188 and Gremophor RH40 were as main factors to the dissolution of the indexes, selecting the best prescription with RSM.

\section{Gremophor RH40 Single Factor}

Based on the of preliminary experiments tablet were granulated and prepared followed by the method under "3.2" using the ratio of Cur: Poloxamer 188: Gremophor RH40 with 1: 5: 0.5, 1: 5: 1 and 1: 5: 1.5 , respectively. When the amount of Gremophor RH40 was 0.5 times of Cur, the granules could be screened, but still caused tablets sticking phenomenon. When the amount of Gremophor RH40 was 1.0 and 1.5 times of Cur, it sieves difficultly and sticks seriously. The results showed that with the increase of the proportion of Gremophor RH40, it was more difficult to screen and illustrated more serious to stick. The amount of Gremophor RH40 in this preparation should be less than half of Cur.

\section{Poloxamer188 Single Factor Test}

On the basis of preliminary experiments, according to the method under " 3.2 " to granulate and prepare tablets, when Cur: Poloxamer 188: Gremophor RH40 ratio were 1: 1: 0.4, 1: 3: 0.4, 1: 5: 0.4 and 1: 7: 0.4, respectively. The dissolution was used as the indicator and tablet sticking situation was compared. When the amount of Poloxamer188 was 3, 5 and 7 times of prescription amount of Cur, the dissolution at $45 \mathrm{~min}$ were $69.40 \%, 64.63 \%, 87.43 \%$ and $97.86 \%$, respectively; While at $120 \mathrm{~min}$, the in vitro dissolution was $85.34 \%, 80.22 \%, 89.71 \%$ and $99.43 \%$, respectively. Dissolution of the ratio 1: 5 and 1: 7 indicated better, but the phenomenon of sticking became more serious with the increase of the amount of Poloxamer188. Therefore, through the prescription optimization the amount of Poloxamer188 should be less than five times of Cur's.

\section{Response Surface Methodology}

Design-Expert 8 software is used to statistically optimize the formulation to improve in vitro dissolution with RSM. According to the principle of 3-Level design, ratio of P188 to Cur $\left(\mathrm{x}_{1}\right)$ and Gremophor RH40 to Cur $\left(\mathrm{x}_{2}\right)$ were selected as the variables and in vitro dissolution at $120 \mathrm{~min}(\mathrm{y})$ as the dependent variable tested in a 13-run experiment to determine the optimum levels (As shown in Table 1). As shown in Figure 1, response surfaces at 45, 60, 90 and 120min were obtained, which reflected the increasing of $\mathrm{y}$ as $\mathrm{x}_{1}$ and $\mathrm{x}_{2}$ were growing. Because of the hydrotropy of P188 and Gremophor RH40, as $\mathrm{x}_{1}$ and $\mathrm{x}_{2}$ increased, $\mathrm{y}$ increased accordingly, but when the amount of the two came to a certain value, it would affect the viscosity of the tablets, which affected the disintegration and dissolution. The analysis of variance in $120 \mathrm{~min}$ was shown in Table 2. The variance analysis results of Table 2 showed that experimental models had significant differences, quadratic term $\mathrm{x}_{1}{ }^{2}$ has a significant influence on dissolution. The least squares method was used to calculate the optimum ratio of Cur: P188: Gremophor $\mathrm{RH} 40=1: 2.52$ : 0.4 .

Table 1 Response Surface Methodology Analysis

\begin{tabular}{cccc}
\hline \multirow{2}{*}{$\begin{array}{c}\text { Experiment } \\
\text { No. }\end{array}$} & P188/Cur & $\begin{array}{c}\text { Gremophor } \\
\text { RH40/Cur }\end{array}$ & $\begin{array}{c}\text { Dissolution(\%) at } \\
\text { 120[min] }\end{array}$ \\
\cline { 2 - 4 } & $\left(\boldsymbol{x}_{1}\right)$ & $\left(\boldsymbol{x}_{2}\right)$ & $(\boldsymbol{y})$ \\
\hline 1 & 4.0 & 0.4 & 59.79 \\
2 & 2.5 & 0.4 & 94.04 \\
3 & 2.5 & 0.4 & 96.84 \\
4 & 2.5 & 0.3 & 87.36 \\
5 & 2.5 & 0.4 & 90.78 \\
6 & 4.0 & 0.5 & 71.17 \\
7 & 2.5 & 0.5 & 72.80 \\
8 & 1.0 & 0.3 & 56.37 \\
9 & 1.0 & 0.4 & 74.60 \\
10 & 1.0 & 0.5 & 65.61 \\
11 & 2.5 & 0.4 & 90.53 \\
12 & 2.5 & 0.4 & 90.53 \\
13 & 4.0 & 0.3 & 68.94 \\
\hline
\end{tabular}




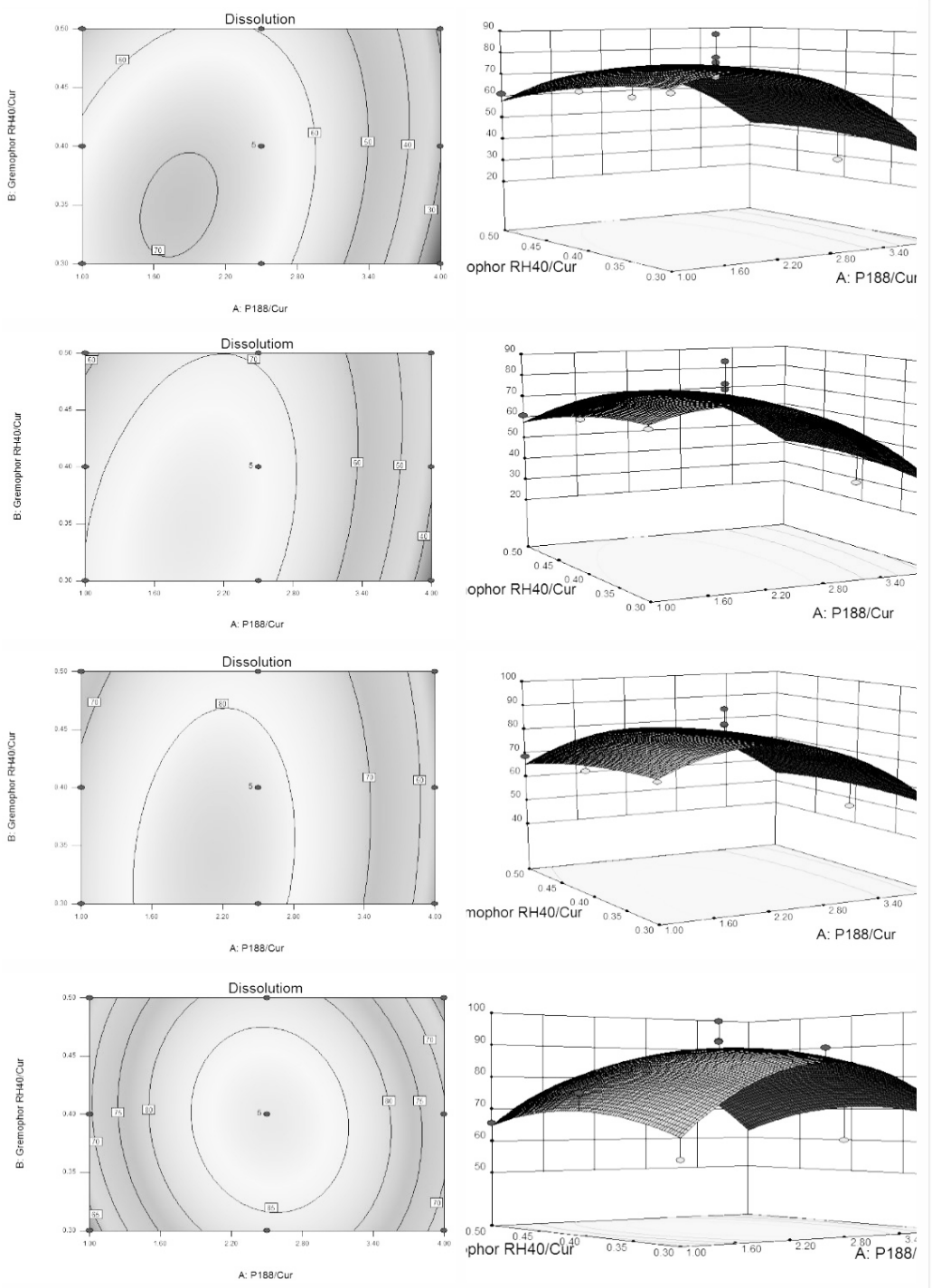

Fig. 1. Response surface renderings (top to bottom are $45,60,90$ and $120 \mathrm{~min}$ response surface map)

Table 2 ANALYSIS of VARIANCE IN 120[min]

\begin{tabular}{lccccc}
\hline Source & $\begin{array}{c}\text { Sum of } \\
\text { Squares }\end{array}$ & $\boldsymbol{d f}$ & $\begin{array}{c}\text { Mean } \\
\text { Square }\end{array}$ & $\boldsymbol{F}$ Value & $\begin{array}{c}\boldsymbol{p} \text {-value } \\
>\boldsymbol{F}\end{array}$ \\
\hline Modle & 1521.81 & 5 & 304.36 & 4.11 & 0.0462 \\
$\boldsymbol{x}_{\boldsymbol{1}}$ & 1.84 & 1 & 1.84 & 0.025 & 0.8792 \\
$\boldsymbol{x}_{2}$ & 1.59 & 1 & 1.59 & 0.021 & 0.8876 \\
$\boldsymbol{x}_{\boldsymbol{1}} \boldsymbol{x}_{\boldsymbol{2}}$ & 12.31 & 1 & 12.31 & 0.17 & 0.6956 \\
$\boldsymbol{x}_{\boldsymbol{1}}{ }^{2}$ & 965.66 & 1 & 965.66 & 13.04 & 0.0086 \\
$\boldsymbol{x}_{2}$ & 93.233 & 1 & 93.23 & 1.26 & 0.2989 \\
Residual & 518.38 & 7 & 74.05 & & \\
Lack of Fit & 371.46 & 3 & 123.82 & 3.37 & 0.1355 \\
Pure Error & 146.93 & 4 & 36.73 & & \\
Cor Total & 2040.19 & 12 & & & \\
\hline
\end{tabular}




\section{Verification Test}

Press optimal formulation, In accordance with the method under "3.2", three batches of curcumin tablets were prepared. The in vitro dissolution results remained the same, the average dissolution was $64.85 \%$ at $45 \mathrm{~min}$ and $95.12 \%$ at $120 \mathrm{~min}$.

\section{The Dissolution of Different Formulations}

Cur pharmaceutical raw materials by count of $10 \mathrm{mg} / \mathrm{cup}$, prescriptions Cur raw materials mixture with the $200 \mathrm{mg}$, capsules of Cur powders and a commercial operator by $10 \mathrm{mg}$ Cur were accurately weighed respectively. The dissolution was measured according to the dissolution procedure. The dissolution of Cur substance, raw materials and accessories prescriptions Cur mixture, capsule powder were $28.31 \%, 29.30 \%, 77.49 \%$ at $45 \mathrm{~min}$ and $46.88 \%, 41.92 \%, 89.21 \%$ at $120 \mathrm{~min}$. The dissolution of Cur tablets by the response surface design of optimal prescription obtained was $64.85 \%$ at $45 \mathrm{~min}$, and $95.12 \%$ at $120 \mathrm{~min}$. The results showed that the tablets prepared along the optimal prescription had higher dissolution compared with raw Cur and physical mixtures, capsule powder.

\section{Conclusions}

Microporous membranes used in this experiment is a polyether sulfone material, curcumin has certain adsorption, membrane adsorption in the experiment, and the filtrate was discarded early $2 \mathrm{ml} 3 \mathrm{ml}$, membrane adsorption amount is less than the average $2 \%$, but the beginning of the filtrate was discarded $3 \mathrm{~mL}$ adsorption filters less experimental error is smaller, so this was chosen $3 \mathrm{ml}$ beginning of the filtrate was discarded.

The study found Gremophor RH40 sample order of dissolution Cur sheet impact, will Gremophor RH40 was adjusted to Cur added prepared curcumin tablets dissolution repeatable, and the corresponding dissolution also increased. Another of the experiment the amount of PVPP screening results show that, PVPP dosage of curcumin on in vitro dissolution of little effect.

Curcumin poorly soluble drugs, for poorly soluble drugs, its dissolution time than the body of drug absorption time, and the dissolution rate is the rate-limiting step of drug absorption. So, dissolution rate and bioavailability have a high correlation [9]. This insoluble drug Cur solubility is not high in ethanol, and much higher in acetone. Thus, acetone was used in the experiment. In order to improve the bioavailability of the drug, this experiment optimized formulation and technology to improve dissolution in vitro of Cur. The preparation process for equipment less demanding, simple operation, eliminating the solid dispersion is heated to melt and freeze-dried preparation to join the cumbersome extra step adhesives and the like, easy to implement industrial production.

\section{References}

[1] LIU A C, LOU H X, ZHAO L X,et al. J Pharm Biomed Anal, 2006, 40 (3): 720-727.

[2] SHIN D H, SEO E Y, PANG B, et al. J Pharma-col Sci, 2010, 115:144-154.

[3] Xiumei Ke, Rongping Yang, Xuming Liang. Natural Product Research and Development, 2013, 25(08): 1154-1159. (In Chinese)

[4] Qi Lili, Wang Jinbo. Food Industry Technology, Food Additives, 2007, 28 (01): 181-182. (In Chinese)

[5] Hui Zhong, Zhu source, Yu Jiangnan etc. Chinese Materia Medica, 2014, 39(17): 3226-3231. (In Chinese)

[6] Linlin Zhao, Han Gang, Zhu Ying. Inner Mongolia in Medicine, 2009, 28(10): 1006-0979. (In Chinese)

[7] Binhui Wang, Wenhong Zhang, Xiaofen Zhang. Chinese Medicine, 2014, 32 (6): 1430-1432. (In Chinese)

[8] Chenchen Li, Feirong Gong, Shujun Cheng. East China University of Technology: Natural Science Edition, 2014, 40 (5): 18-23. (In Chinese) 
[9] Royal Hanson, Vivian Gray, Baoming Ning. Dissolution Testing and other Technology, M. Beijing: Chinese Medical Science and Technology Publishing House, 2007. (in Chinese)

[10] Wang Yamin. Journal of Pharmaceutical Analysis, 2007, 27 (10): 1667-1670. (In Chinese)

[11] Mufeng Xie. Chinese Journal of Industrial Medicine, 2012, 43 (3): A23-A32. (In Chinese)

[12] Sheskey (US), Weller (Britain), Junmin Zheng. Pharmaceutical Excipients Manual, M. China: Chemical Industry Press, 2005.548-553. (In Chinese) 